\title{
Surgical and visual outcome of posterior polar cataract
}

\author{
Pooja. H.V., ${ }^{1 *}$, H. T. Venkate Gowda ${ }^{2}$ \\ ${ }^{1}$ Senior Resident, ${ }^{2}$ Professor \& HOD, Dept. of Ophthalmology, Adichunchanagiri Institute of Medical Sciences, Mandya district, \\ Karnataka, India
}

*Corresponding Author:

Email: poojahv1410@gmail.com

\begin{abstract}
Purpose: To evaluate the surgical and visual outcome of manual small incision cataract surgery in patients with posterior polar cataract.

Material and Methods: A prospective study was conducted from June 2018 to August 2018. 26 eyes of 26 patients underwent surgery at our hospital were included in our study. All patients underwent complete ophthalmologic examination which included visual acuity, slit-lamp biomicroscopy, IOP and fundoscopy. Patients with complicated cataract, glaucoma, corneal opacity/ degeneration, retinal pathology were excluded. Patients were scheduled for manual SICS. Informed consent was obtained from all the patients.

Results: 26 eyes of 26 patients were included in the study. There were 19 males (73.07\%) and 7 females (26.92\%). The mean age was 53.5 (range 37-70) years. The most common intraoperative complication was posterior capsule rupture, seen in 3 eyes $(11.53 \%)$. The intraocular lenses were implanted in the sulcus in those 3 patients and rest in the bag implantation was done. 1 eye needed anterior vitrectomy. The mean visual acuity improved significantly after surgery.

Conclusion: Posterior polar cataract is a great challenge for cataract surgeons. The visual outcome is good if done carefully with proper technique advised for PPC.
\end{abstract}

Keyword: PPC, PCR, Vitrectomy.

\section{Introduction}

Posterior polar cataract (PPC) is a relatively uncommon form of congenital cataract. The incidence of Posterior polar cataract ranges from 3 to 5 in $1000 .^{1,2}$ It is bilateral in $65-80 \%$ of the cases with no gender predilection. ${ }^{3}$ Posterior polar cataracts poses a great challenge to the surgeons, because of associated complications like posterior capsular rupture, vitreous loss and possible nucleus drop during surgery. The adherence of the plaque to a normal posterior capsule (PC) leads to breach in PC during cortical wash. Also the thin PC can rupture during hydro procedures. DukeElder classified it as stationary and progressive forms, ${ }^{4}$ of which stationary form is more common (about 65\%), Schroeder $^{5}$ graded posterior polar cataract in his pediatric patients based on its effect on pupillary obstruction in the red reflex. Singh classified posterior polar cataract into2: Type 1: The PPC with posterior subcapsular cataract. Type 2: Sharply defined round or oval opacity with onion ring appearance with or without grayish spots at the edge. Type 3: Sharply defined round or oval white opacity with dense white spots at the edge often associated with thin or absent PC. These dense white spots are a diagnostic sign (Daljit Singh sign) of posterior capsule leakage and extreme fragility. Type 4: combination of above 3 types with nuclear sclerosis.

\section{Material and Methods}

A prospective study was conducted from June 2018 to August 2018 at our tertiary care hospital. 26 eyes of 26 patients with posterior polar cataract underwent surgery at our hospital were included in our study. Patients with corneal opacity/ degeneration, complicated cataract, glaucoma, retinal pathology were excluded from the study. Patients typically had symptoms like increased glare while driving at night and difficulty in reading fine prints. All patients underwent complete ophthalmologic examination which included visual acuity, slit-lamp biomicroscopy, IOP and fundoscopy. Slit lamp biomicroscopy revealed disc shaped posterior lens opacity, adjacent to the posterior capsule. Written informed consent was obtained after explaining possible complications like posterior capsule rupture, a relatively long operative time, possible requirement of secondary posterior segment intervention, possibility of preexisting amblyopia, and delayed visual recovery. Patients were scheduled for manual small incision cataract surgery (SICS). After routine preparation, all surgeries were carried out under peribulbar anaesthesia. All surgeries was performed by single surgeon. In SICS, after routine preparation, a frown shaped scleral incision was made. A relatively larger continuous curvilinear capsulorrhexis of about $6 \mathrm{~mm}$. Hydrodissection was avoided in all cases and careful hydrodelineation was performed. Nuclear was delivered by viscoexpression technique. Cortex and epinucleus were aspirated using simcoe cannula. PCIOL was placed in the bag or sulcus depending on the case. Postoperatively was started on topical antibiotic- steroid drops. All the data will be analyzed using frequency and percentage. 


\section{Results}

26 eyes of 26 patients were included in the study. There were 19 males $(73.07 \%)$ and 7 females $(26.92 \%)$. The mean age was 53.5 (range 37-70) years. The most common intraoperative complication was posterior capsule rupture, seen in 3 eyes $(11.53 \%)$. The intraocular lenses were implanted in the sulcus in those 3 patients and rest $23(88.46 \%)$ in the bag implantation was done.1 (3.84\%) eye needed anterior vitrectomy. The mean visual acuity improved significantly after surgery. Uncorrected Visual acuity (UCVA) of 6/18 or better was seen in 2 eyes $(7.69 \%)$ preoperatively. (Table 1) UCVA of $6 / 18$ or better after surgery was seen in a total of 19 eyes $(73.07 \%)$. Only one eye had UCVA of < 6/60 due to corneal oedema which improved to $6 / 18$ on conservative management. (Table 2)

Table 1

\begin{tabular}{|l|l|}
\hline Preooperative UCVA & \multicolumn{1}{c|}{ Total } \\
\hline $6 / 6-6 / 18$ & $2(7.69 \%)$ \\
\hline$<6 / 18-6 / 60$ & $4(15.38 \%)$ \\
\hline$<6 / 60-3 / 60$ & $17(65.38 \%)$ \\
\hline$<3 / 60-\mathrm{HM}$ & $3(11.53 \%)$ \\
\hline & $26(100 \%)$ \\
\hline
\end{tabular}

Table 2

\begin{tabular}{|l|c|}
\hline Postoperative UCVA & Total \\
\hline $6 / 6-6 / 18$ & $19(73.07 \%)$ \\
\hline$<6 / 18-6 / 60$ & $6(23.07 \%)$ \\
\hline$<6 / 60-3 / 60$ & $1(3.84 \%)$ \\
\hline$<3 / 60-\mathrm{HM}$ & - \\
\hline & $26(100 \%)$ \\
\hline
\end{tabular}

\section{Discussion}

Posterior polar cataract is a challenge for the cataract surgeon as these are associated with higher complication rate like PCR $(0-40 \%)$ and vitreous loss. ${ }^{6-}$ ${ }^{8}$ In various studies the incidence of posterior capsule rupture has been reported to be $26 \%^{3}$ and $36 \% .^{6}$ In contrast, posterior capsule rupture was noted in only $11.53 \%$ of our study. Vasavada AR et $\mathrm{al}^{9}$ and Hayashi $\mathrm{k}$ et $\mathrm{al}^{10}$ reported $4 \%$ incidence of nucleus drop. In our study none of the patients had nucleus drop. Visual acuity improved significantly in our study which is shown in other study as well. ${ }^{10}$ Difficulty in carrying out daily activities is the main indication for surgery. Because of the possibility of the formation of capsular defects over time, early intervention is required. Also there is a possibility of amblyopia in visually significant cataract since childhood.

\section{Conclusion}

The incidence of preexisting amblyopia should be noted in patients with unilateral posterior polar cataract. Posterior polar cataract is a great challenge for cataract surgeons. The visual outcome is good if done carefully with proper technique advised for PPC.

Funding: No funding sources.

Conflict of interest: None declared.

\section{References}

1. Lee MW, Lee YC. Phacoemulsification of posterior polar cataracts - a surgical challenge. $\mathrm{Br} \quad \mathrm{J}$ Ophthalmol 2003;87:1426-27.

2. Masket S. Consultation section: Cataract surgical problem. J Cataract Refract Surg 1997;23:819-24.

3. Osher RH, Yu BC, Koch DD. Posterior polar cataracts: a predisposition to intraoperative posterior capsular rupture. J Cataract Refract Surg 1990;16:157-62.

4. Duke-Elder S. Congenital deformities. Part 2. Normal and Abnormal Development. System of Ophthalmology; vol. III. St. Louis: CV Mosby; 1964.

5. Schroeder HW. The management of posterior polar cataract: the role of patching and grading. Strabismus 2005;13(4):153-56.

6. Vasavada AR, Singh R. Phacoemulsification with posterior polar cataract. J Cataract Refract Surg 1999;25:238-45.

7. Skalka HW. Ultrasonic diagnosis of posterior lens rupture. Ophthalmic Surg 1977;8(6):72-6.

8. Hiles DA, Chotiner B. Vitreous loss following infantile cataract surgery. J Pediatr Ophthalmol 1977;14:193-99

9. Vasavada A, Singh R. Phacoemulsification in eyes with posterior polar cataract. J Cataract Refract Surg 1999;25:238-45.

10. Hayashi K, Hayashi H, Nakao $F$ et al. Outcomes of surgery for posterior polar cataract. $J$ Cataract Refract Surg 2003;29:45-9.

How to cite the article: H. Pooja, Gowda V. Surgical and visual outcome of posterior polar cataract. Int $J$ Ocul Oncol Oculoplasty 2018;4(3):138-139. 